\title{
The doctor and the mask: iatrogenic septic arthritis caused by Streptoccocus mitis
}

\author{
A 72-year-old man developed septic arthritis in a prosthetic shoulder after intra- \\ articular injection of radiographic contrast. This is the first published case in which \\ molecular techniques matched oral commensal organisms cultured from joint \\ aspirate with oral flora from the proceduralist, who was not wearing a mask.
}

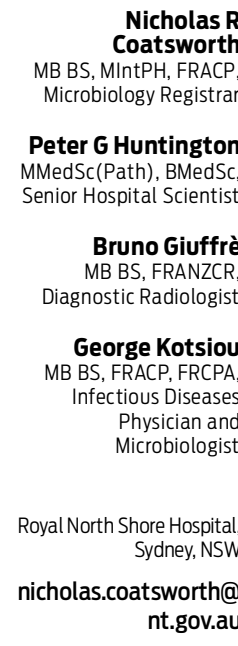

doi: $10.5694 / \mathrm{mjal2.11695}$

\section{Clinical record}

A 72-year-old man presented in 2011 with acute-on-chronic right shoulder pain. Bilateral shoulder replacements had been performed 8 years earlier for osteoarthritis, with no surgical complications.

In the 6 months before presentation, the patient experienced increasing pain and decreased range of movement of the right shoulder. Four days before presentation, a computed tomography (CT) arthrogram of the right shoulder was performed to look for glenoid osteolysis and assess the linear integrity of the shoulder prosthesis. Eight millilitres of radiographic contrast with bupivacaine were injected into the joint space. The proceduralist used an aseptic technique and skin preparation with $0.5 \%$ alcoholic chlorhexidine, but did not wear a mask. Within 24 hours, the shoulder pain dramatically worsened and the range of movement became severely impaired.

The patient had a history of hypertension, severe obstructive sleep apnoea, and paroxysmal atrial fibrillation. He was taking indapamide, perindopril and warfarin, and used nocturnal continuous positive airway pressure. In 1993, he had been treated with radiotherapy for prostate cancer and was taking finasteride.

On presentation, the patient was febrile $\left(38.4^{\circ} \mathrm{C}\right)$. Clinical examination showed a warm, swollen right shoulder and pain on passive movement of the joint. The C-reactive protein level was elevated at $229 \mathrm{mg} / \mathrm{L}$ (reference interval, $<5 \mathrm{mg} / \mathrm{L})$.

Ultrasound-guided aspiration of the joint recovered a highly inflammatory fluid with a white cell count of $174.6 \times 10^{9} / \mathrm{L}$, and $1+$ gram-positive cocci were identified. Arthroscopic washout was performed, and the patient was given intravenous flucloxacillin $2 \mathrm{~g}$ every 6 hours and benzylpenicillin $1.8 \mathrm{~g}$ every 6 hours.

Culture yielded light growth on the primary plates of Streptococcus mitis group 1 and scant Haemophilus parainfluenzae. The antibiotic dose was changed to intravenous benzylpenicillin $2.4 \mathrm{~g}$ every 6 hours with synergistic gentamicin $240 \mathrm{mg}$ daily for the first 2 weeks. After 4 weeks of intravenous therapy, the patient was switched to oral amoxicillin $1 \mathrm{~g}$ three times daily to complete a 3-month course of antibiotics.

Shortly after the patient presented, the proceduralist agreed to provide an oropharyngeal swab. Several organisms were cultured, including multiple viridans Streptococcus species. Pulsed-field gel electrophoresis (PFGE) was performed according to the method of Lefevre et al, with modifications. ${ }^{1}$ Using two different restriction enzymes (SmaI, ApaI), we found that the patient's organism and a strain of S. mitis recovered from the proceduralist's throat showed indistinguishable fragment patterns (Box). This strongly suggested droplet transmission of the proceduralist's oral flora onto the needle or skin, with subsequent inoculation into the shoulder joint.

Six months after the acute presentation, joint failure, confirmed on arthrogram and by arthroscopy, necessitated full revision of the right shoulder prosthesis. The procedure was uncomplicated, and the patient remains well with no signs of recurrent infection.

\section{Discussion}

This is the first published instance of a molecular epidemiology technique showing probable transmission of oral flora from a proceduralist to the joint of a patient, resulting in iatrogenic septic arthritis.

Australian Medicare data for the period 2006-2009 show that an average of 516562 claims were made annually for joint injections or aspirations. ${ }^{2}$ The estimated incidence of septic arthritis after intra-articular corticosteroid injection into a native joint is estimated to be between 1 per 3000 and 1 per 16000 injections. $^{3}$ Applying these incidence rates to Australian data, we would estimate that between 30 and 180 instances of iatrogenic septic arthritis per year are a result of joint injection or aspiration. It could be safely assumed that even fewer of these could be attributed to omission of a surgical mask. Given this apparently low burden of disease, should a surgical mask be a mandatory requirement of an aseptic technique for this procedure?

Infection control practices during the injection of sterile sites vary substantially across specialties and depend on the type of procedure and where it is performed (general practice, wards, operating theatres). Several studies confirm the anecdotal evidence that mask-wearing while injecting into sterile sites is not standard practice across a number of specialties, including rheumatology, ${ }^{4}$ obstetric anaesthesia ${ }^{5}$ and general practice. ${ }^{6}$ In some series, the rate is as low as $11 \%$, and a debate exists in the surgical literature about whether surgical masks should be used at all in operating theatres. ${ }^{6}$

However, there is good microbiological evidence that oral bacterial flora, of which oral (viridans) streptococci predominate, can be deposited on an agar plate held at $30 \mathrm{~cm}$ from a speaking subject's mouth for a period of 5 minutes. ${ }^{7}$ Thus, if a mask is omitted, procedures such as spinal anaesthesia, or any teaching procedure that can involve speaking to the 
1 Pulsed-field gel electrophoresis dendrogram: Streptococcus mitis isolates from the patient's joint fluid (Lane 1), and the proceduralist's oropharynx (Lane 2 and Lane 3 ), showing an indistinguishable restriction fragment pattern. The lanes below are other oral Streptococcus isolates from the proceduralist along with control organisms

$$
20 \quad 40 \quad 60 \quad 80100
$$
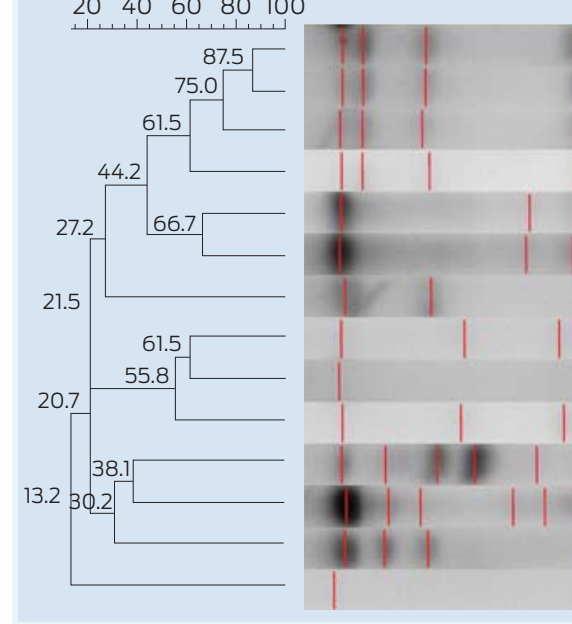

because of the physical configuration of the patient's mouth relative to the sterile field. In addition, the microbiological similarity between iatrogenic septic arthritis and post-lumbar puncture meningitis (where contamination by the patient's oral flora cannot reasonably be asserted) is compelling. Other sources of contamination from patients, such as skin flora or groin organisms, are unlikely given the preponderance of viridans streptococci and the striking absence of gram-negative bacilli and Staphylococcus species. We did not feel it necessary to take a mouth swab from the patient in this case.

Although no reports to date have been able to link a proceduralist's oral flora to the causative pathogen in nosocomial septic arthritis, we consider the transmission of an oral Streptococcus species to a sterile joint space as analogous to the demonstration of identical organisms in iatrogenic meningitis.

Some authors have suggested that the efficacy of surgical masks is unproven, and that viridans streptococci may be introduced in ways other than direct contamination from the oropharynx. ${ }^{14}$ In particular, it has been suggested that transmission could be explained by more general deficiencies in aseptic technique, including contamination of the equipment during set-up or improper skin sterilisation. However, the compelling evidence that oral commensal bacteria can be aerosolised, and molecular confirmation of the source in several cases, including our own, would suggest that a surgical mask serves a role in aseptic technique.

Based on this case, we would recommend that clinicians seek a history of recent joint intervention in circumstances in which viridans streptococci are isolated from joint culture, particularly when the organism grows from the direct inoculum as well as the enrichment medium. We consider a surgical mask to be a low-cost, simple addition to the aseptic technique that may assist in prevention of nosocomial septic arthritis. graph. A review of 179 cases of iatrogenic meningitis provides corroborative evidence that low virulence organisms can be dispersed from the oropharynx to sterile sites and cause infection. ${ }^{9}$ The evidence indicates that the risk of meningitis is far higher when there is inoculation into a sterile site (eg, spinal anaesthesia) than simple needle puncture of the site (eg, lumbar puncture). Similarly, the risk of iatrogenic septic arthritis is likely to be greater with inoculation than aspiration alone. That a low virulence organism can cause such morbidity, and occasionally death from meningitis, likely relates to the breach of usual host immune defences by direct inoculation into the site of infection. On rare occasions molecular confirmation of the source, using either PFGE or polymerase chain reaction, has been documented after recovery of identical organisms from the oropharynx of the proceduralist. $^{10}$

It is always challenging to prove relatedness of bacterial strains, as typing techniques are often dependent on the specific bacterial species. PFGE has been shown to be a reliable technique for differentiating strains of $S$. mitis in other studies. ${ }^{11,12}$ In our case, the use of two different restriction endonucleases with identical results adds robustness to the data. While molecular methods can only ever prove two bacterial strains are different, there are established criteria for relatedness. ${ }^{13}$ The combination of our two pulsedfield gels satisfies the Tenover criteria for indistinguishable strains.

For most procedures, it is highly improbable that oral flora from the patient are the source of contamination, simply

\section{Competing interests: No relevant disclosures.}

Received 19 Nov 2012, accepted 24 Feb 2013.

1 Lefevre JC, Faucon G, Sicard AM, Gasc AM. DNA fingerprinting of Streptococcus pneumoniae strains by pulsed-field gel electrophoresis J Clin Microbiol 1993; 31: 2724-2728.

2 Australian Government Department of Health and Ageing. Medicare Australia Statistics 2013 [updated 25 Jan 2013]. https://www.medicareaustralia.gov.au/cgi-bin/broker.exe?_PROGRAM=sas.mbs_item_standard_ report.sas\&_SERVICE $=$ default\&DRILL=ag\&_DEBUG $=0 \& g r o u p=50124 \% 2 C+50125 \& V A R=$ services\&STAT $=$ count\&RPT_FMT=by+state\&PTYPE=finyear\&START_DT=200607\&END_DT=200906 (accessed Feb 2013).

3 Von Essen R, Savolainen HA. Bacterial infection following intra-articular injection: a brief review. Scand J Rheumatol 1989; 18:7-12.

4 Haslock I, Macfarlane D, Speed C. Intra-articular and soft tissue injections: a survey of current practice. Br J Rheumatol 1995; 34: 449-452.

5 Panikkar KK, Yentis SM. Wearing of masks for obstetric regional anaesthesia: a postal survey. Anaesthesia 1996; 51: 398-400.

6 Davis P, Spady D, Forgie SE. A survey of Alberta physicians' use of and attitudes toward face masks and face shields in the operating room setting. Am J Infect Control 2007; 35: 455-459.

7 Philips BJ, Fergusson P, Armstrong P, et al. Surgical face masks are effective in reducing bacterial contamination caused by dispersal from the upper airway. Br J Anaesth 1992; 69: 407-408.

8 Armstrong RW, Bolding F, Joseph R. Septic arthritis following arthroscopy: clinical syndromes and analysis of risk factors. Arthroscopy 1992; 8: 213-223.

9 Baer ET. Post-dural puncture bacterial meningitis. Anesthesiology 2006; 105: 381-393.

10 Veringa E, Van Belkum A, Schellekens H. latrogenic meningitis by Streptococcus salivarius following lumbar puncture. J Hosp Infect 1995; 29: 316-318.

11 Wisplinghoff H, Reinert RR, Cornely O, Seifert H. Molecular relationships and antimicrobial susceptibilities of viridans group streptococci isolated from blood of neutropenic cancer patients.J Clin Microbiol 1999; 37: 1876-1880.

12 Lu H, Weng X, Zhu B, et al. Major outbreak of toxic shock-like syndrome caused by Streptococcus mitis. J Clin Microbiol 2003; 41: 3051-3055.

13 Tenover FC, Arbeit RD, Goering RV, et al. Interpreting chromosomal DNA restriction patterns produced by pulsedfield gel electrophoresis: criteria for bacterial strain typing. J Clin Microbiol 1995; 33: 2233-2239.

14 Black SR, Weinstein RA. The case for face masks - Zorro or zero? Clin Infect Dis 2000; 31: 522-523. 